\title{
Improving production data quality in underground operations
}

N Ferreira Modular Mining Systems, Inc., USA

\begin{abstract}
One of the challenges in underground mining is a lack of operational visibility. By implementing a fleet management system (FMS) designed for the underground environment, mines are able to determine where their mobile equipment is located, what it is doing, and how it is performing, at all times. In addition, communication challenges underground can often make it difficult to capture reliable production metrics needed for effective decision-making. Examples include equipment cycle times, distance travelled, quantity and qualities of material moved, orepass inventory, work place status, etc.

An effective FMS addresses all major aspects of the development and production processes, and provides a solution that manages the numerous and ongoing sequential, concurrent, and parallel efforts present in underground mining. With the right FMS, mines can realise increased equipment utilisation and productivity, maximised material movement, reduced waste and operational costs, and enhanced operator safety.
\end{abstract}

This paper will demonstrate how an underground FMS helped an operation improve data accuracy through production cycle automation, offline data capture, and automated communications network switching.

Keywords: visibility, automation, underground, fleet management

\section{Introduction}

Underground mining presents some unique challenges not encountered by surface mining operations, including the lack of operational visibility and limited communication infrastructure. Mine operators can struggle with finding answers to the most basic questions:

- Where is my equipment and what is it doing?

- What is my current production rate?

- How am I progressing towards the mine plan?

This lack of operational visibility and reliable data capture can result in poor decision-making and a less optimised workflow. With a comprehensive underground FMS, mines can improve efficiency, equipment utilisation, and production reporting accuracy, through the use of automated task management, equipment location and status tracking, as well as real-time data capture.

\section{Opportunities from underground fleet management systems}

Underground FMS configurations can range from simple voice-based tracking systems, where equipment operators periodically call into a central control room to report on the status of their work, to automated real-time data capture solutions that reduce the reliance on operator input, resulting in improved data quality.

Day-to-day operations can be enhanced through the underground FMS's ability to provide visibility into the areas of:

- Fleet management.

- Production task monitoring.

- Equipment time tracking. 
- Equipment location.

- Crew management.

○ Scheduling.

- Operator qualifications.

- Key performance indicators.

- Material movement.

- Material classification.

- Material quantity tracking.

- Material quality tracking.

- Inventory management.

- Real-time inventory tracking.

- Average grade and density tracking.

- Survey adjustments.

By effectively managing the three key areas shown below, underground operations can improve overall asset utilisation, resulting in greater production capacity. The visibility that the FMS provides may help mines to focus on the key operational areas shown in Figure 1, and enable mines to better track equipment location, manage crew and equipment activity and performance, and assess production progress and adherence to plan.

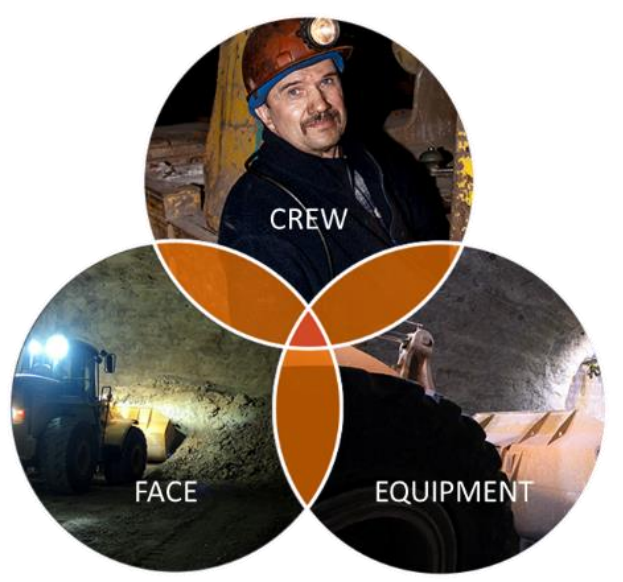

Figure 1 Key focus areas

\section{Case study}

Managing draw control in block cave operations is critical to the success of the cave. Poor draw practices can increase dilution, raise the risk of air-blasts and mud-rushes, reduce fragmentation, and ultimately halt cave propagation, potentially resulting in lower productivity and decreased production rates. The accurate recording and analysis of the tonnages drawn from each drawpoint is crucial to effective draw control and is often not given the respect it requires in the overall mining process.

A large Chilean block cave operation, which utilises the DISPATCH ${ }^{\circledR}$ Underground FMS to record draw quantities, contracted Modular Mining Systems to conduct an audit of the current processes to identify areas for improvement in their draw control process and FMS usage. The baseline process workflow was determined to be as follows: draw orders, in the form of a draw card, are issued every shift from a computerised cave management system (CMS). The draw orders are uploaded to the on-board FMS field computer as work instructions for load-haul-dump (LHD) operators. Actual tonnages drawn from each 
drawpoint are automatically recorded by the FMS based on the LHD operators manually selecting the extraction drawpoint on the field computer. Actual draw card production data is transferred to the CMS at the end of the shift so that new draw orders can be computed based on the over or under-tonnage draw from each drawpoint. A new draw order is then issued for the next shift.

\subsection{Baseline state}

Production data captured for the month of December 2014 was analysed. The data consisted of semi-automatic load counts from eight production LHD units, as well as load data entered into the system by the dispatchers in the control room based on LHD operators' paper records. The equipment operators manually tracked the loads completed at each drawpoint. By comparing the number of automatically captured loads with the number of manually captured loads recorded by the operators, the mine could determine how many loads the dispatchers needed to manually enter into the DISPATCH FMS. When the process was complete, the FMS would have a record of all completed loads, by drawpoint.

The manual data captured accounted for $15 \%$ of the total production; an amount equal to 169,881 loads, as show in Figure 2.

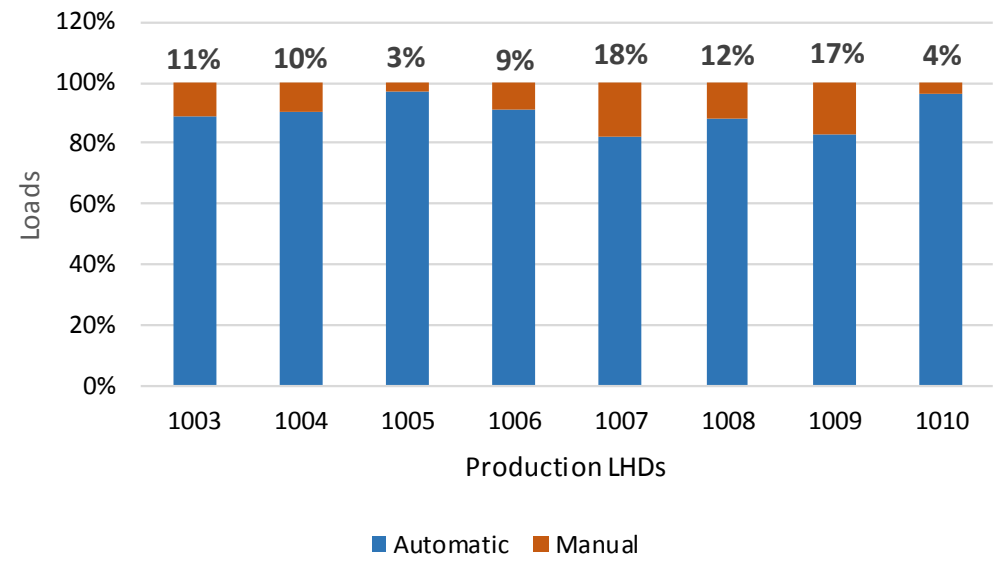

Figure 2 December 2014 production data

The large number of manual loads recorded was of concern, as this directly affects the level of confidence in the effectiveness of the draw control. Manual data recording is subject to human error, which can lead to unrecorded loads or misrepresentation of actual load values.

Further analysis of the cause of the high manual load count determined that the field computers in use at the mine were nearly 20 years old and had no on-board data storage. As a result, the field computers were unable to capture data during communications outages. In addition, the mine's distributed antenna system (DAS) infrastructure failed to provide communication coverage in all working areas.

\subsection{Remedial action}

Upon conclusion of the audit, conducted by the mining services division, Modular Mining Systems Chile, the mine received the following recommendations for improving overall production data collection:

- Upgrade all field computers to the latest generation hardware. Doing so would enable the field computers to capture and store production data locally, via the DISPATCH system's Store\&Forward feature, while the equipment is out of communication range. When communications are re-established, the stored data is immediately forwarded to the DISPATCH FMS's central server where events are automatically processed and integrated with existing production records, in real time.

- Increase communication coverage in the active mining areas. 


\subsubsection{Phase 1}

The mine upgraded all mobile equipment field hardware to Modular's current-version PTX Mobile Hardware platform. In addition to enabling the Store\&Forward feature for offline draw order management, this platform also facilitated automatic draw order management.

The DISPATCH Underground FMS automates the equipment production cycle data capture to facilitate accurate material quantity and quality movement records, equipment time tracking, task assignments, and task progress. This is accomplished by outfitting production equipment units with ruggedised field computers, radiofrequency identification (RFID) tag readers, and data radios. RFID tags placed in strategic locations within the tunnel network facilitate equipment movement tacking and production cycle automation.

\subsubsection{Draw order management}

Implementation of automatic haul cycle state transitions based on RFID positioning eliminated the mine's reliance on equipment operators' manual input to select the extraction drawpoint for the load count. The auto-haul cycle functionality was coupled with a new on-board draw card feature that automatically tracked the loads taken from each drawpoint. The LHD equipment did not have payload systems to provide actual tonnage. The standard bucket factor methodology was used to convert loads to tons.

The new draw card application presented the drawpoint ID, planned loads, actual loads, and remaining loads, to the operator. Colour coding was included for easy visual recognition of drawpoints that were on target, underdrawn, or overdrawn, as shown in Figure 3.

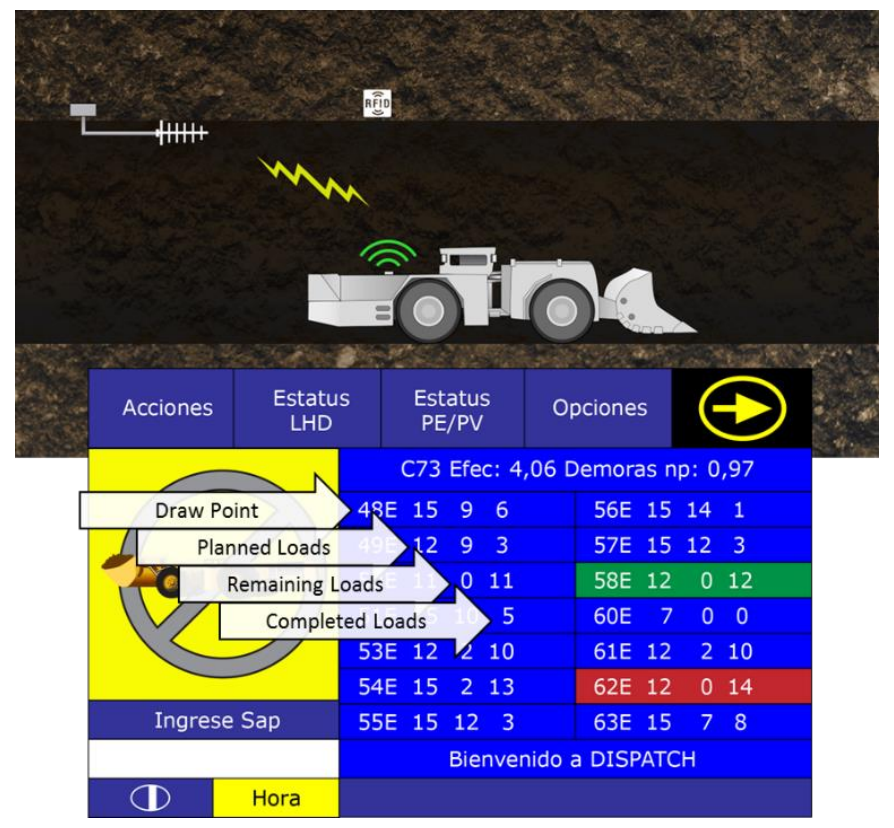

Figure 3 On-board draw card

\subsubsection{Phase 1 results}

At the end of Phase 1, the mine experienced a significant improvement in the number of automatic loads captured as the result of the Store\&Forward and haul cycle automation capabilities provided by the DISPATCH Underground system.

Manual data adjustments accounted for only $4 \%$ of total production; a $76 \%$ reduction in manual load adjustments and a $14 \%$ overall improvement in the automatic load capture over the 2014 baseline, as shown in Figure 4 and Table 1. 


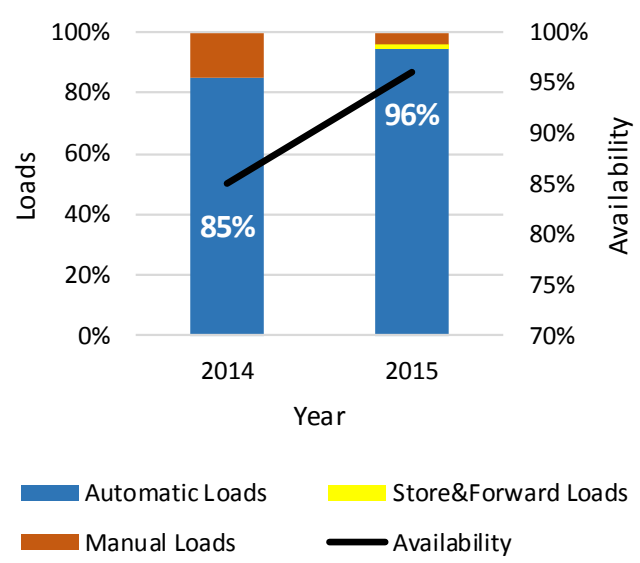

Figure 4 Automatic versus manual loads 2014-2015

Table 1 Percentage difference in automatic versus manual loads 2014-2015

\begin{tabular}{llllll}
\hline & $\mathbf{2 0 1 4}$ loads & $\mathbf{2 0 1 4} \%$ total loads & 2015 loads & $\mathbf{2 0 1 5} \%$ total loads & $\boldsymbol{\Delta} \%$ \\
\hline Manual & 169,881 & 15 & 41,491 & 4 & 76 \\
Offline & 0 & NA & 18,892 & 2 & NA \\
Online & 965,889 & NA & $1,083,036$ & 95 & 12 \\
Automatic & 965,889 & 85 & $1,101,928$ & 88 & 14 \\
Total loads & $1,135,770$ & 85 & $1,143,419$ & NA & 1 \\
\hline
\end{tabular}

Note: $\Delta=$ difference

\subsubsection{Phase 2}

Before the audit, the mine had embarked on a program to supplement its DAS infrastructure with Wi-Fi coverage in the underground workshop areas. In Phase 2, the mine extended Wi-Fi coverage into the main access travel ways in the new mining areas. In addition, the field computer mobile application was enhanced to support multiple data radios with automatic switching between Wi-Fi and DAS, based on network availability.

\subsubsection{Phase 2 results}

Marginal improvement was made in the number of automatic loads recorded, however, because the equipment could automatically select the best quality communications network, dependency on the Store\&Forward functionality was reduced, accounting for less than $0.5 \%$ of the total loads, as shown in Figure 5 .

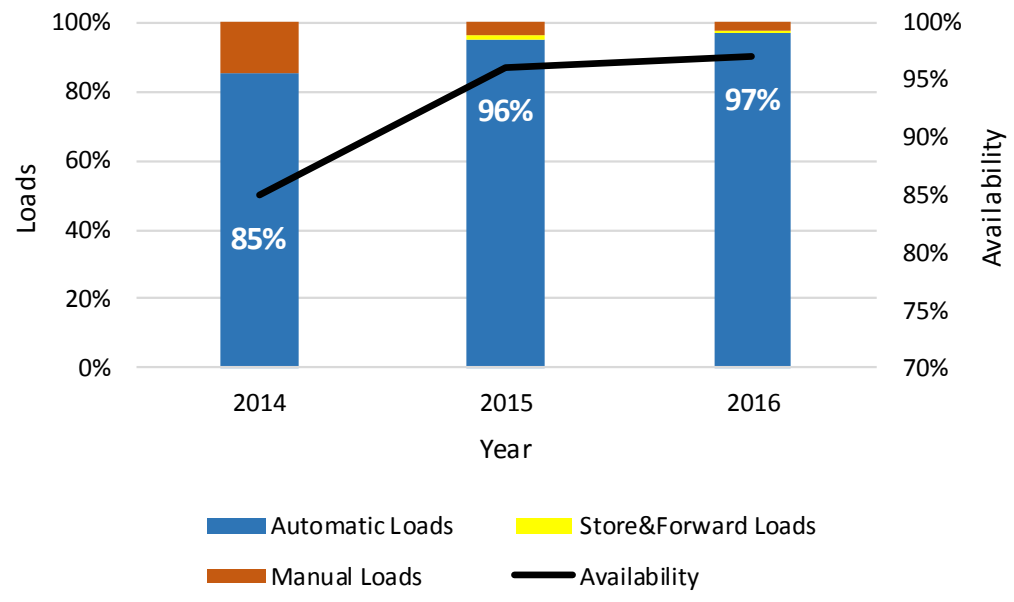

Figure 5 Automatic versus manual loads 2014-2016 
The individual variance between manual and automatic load counting per LHD equipment units is shown in Figure 6.

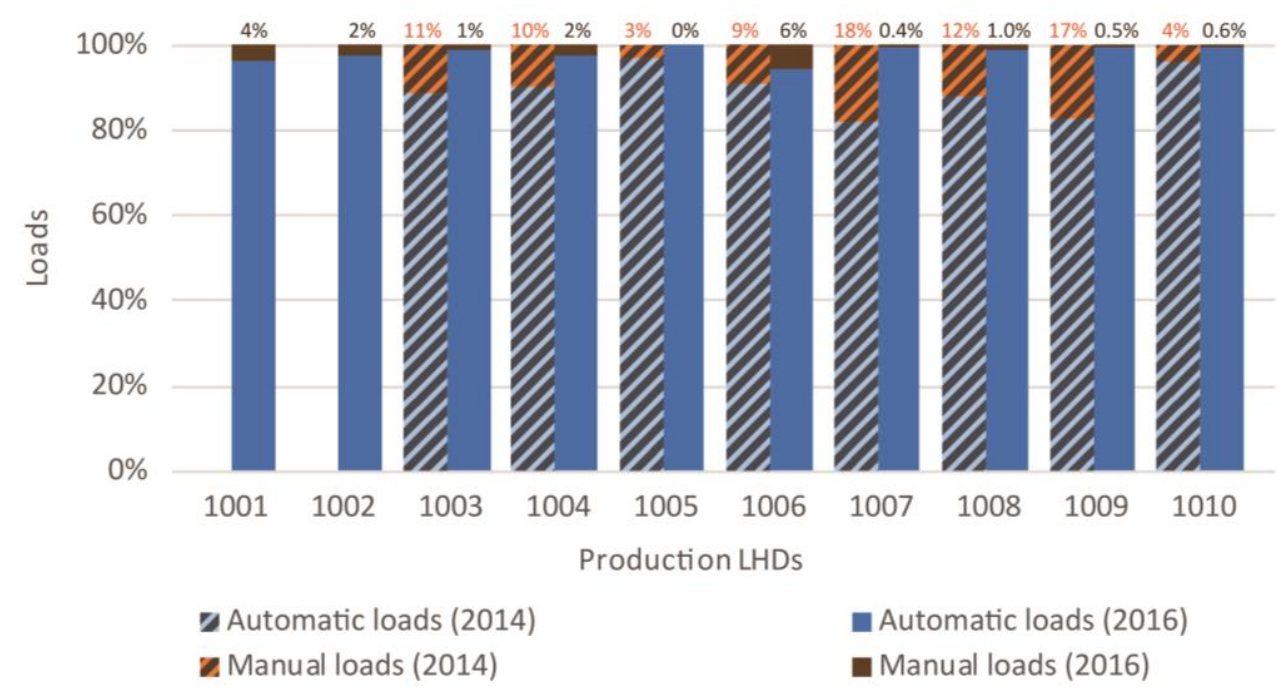

Figure 6 December 2014 versus June 2016 production data

\section{Conclusion}

Despite damaged RFID tags in the drawpoints, which necessitated manual load counting in the affected areas, offline data capture and automatic network switching facilitated by the FMS resulted in a reliable production accounting improvement from 85 to $97 \%$. The new mobile interface provided visual feedback to the operators on the status of the tag reader and displayed the cycle state transitions; a positive indication that the tags were being read correctly. This significant improvement in the automatic capture of production data and resultant reliable feedback provided to the CMS resulted in higher confidence in the control of the block cave propagation. 